\title{
Natural orbits of atomic Cooper pairs in a nonuniform Fermi gas
}

\author{
Y. H. Pong and C. K. Law \\ Department of Physics and Institute of Theoretical Physics, \\ The Chinese university of Hong Kong, Shatin, Hong Kong SAR, China
}

(Dated: November 19, 2018)

\begin{abstract}
We examine the basic mode structure of atomic Cooper pairs in an inhomogeneous Fermi gas. Based on the properties of Bogoliubov quasi-particle vacuum, the single particle density matrix and the anomalous density matrix share the same set of eigenfunctions. These eigenfunctions correspond to natural pairing orbits associated with the BCS ground state. We investigate these orbits for a Fermi gas in a spherical harmonic trap, and construct the wave function of a Cooper pair in the form of Schmidt decomposition. The issue of spatial quantum entanglement between constituent atoms in a pair is addressed.
\end{abstract}

PACS numbers: 03.75.Ss, 05.30.Fk, 74.20.Fg, 03.67.Mn 


\section{INTRODUCTION}

Quantum degenerate gas of two-component Fermi atoms provides a well-controlled system for studying Cooper pairing responsible for superfluidity phenomena in the BCS regime 1, 2]. With the advances in cooling and trapping techniques, recent experiments have demonstrated various consequences of pairing [3, 4, 5, 6]. One of the major tasks for theoretical investigation is the structure of Cooper pairs in non-uniform finite systems. Since the weakly interacting ultra-cold atoms mainly involve two-body $s$-wave scattering processes, detailed analysis can be performed via the pseudo-potential method and Bogoliubov-deGennes (BdG) equations [7, 8]. An important quantity that can be obtained self-consistently by this approach is the gap function. Such a quantity measures the pairing field at a given point in space, but it lacks the information about correlation between atoms at different spatial points. In order to gain a more complete picture of pairing, it is useful to examine properties of two-point correlation functions $[9]$.

In this paper we examine the underlying mode structures inherent in two-point correlation functions: $\left\langle\psi_{\alpha}^{\dagger}\left(\mathbf{r}_{1}\right) \psi_{\alpha}\left(\mathbf{r}_{2}\right)\right\rangle$ and $\left\langle\psi_{\beta}\left(\mathbf{r}_{1}\right) \psi_{\alpha}\left(\mathbf{r}_{2}\right)\right\rangle$, with $\psi_{j}(j=\alpha, \beta)$ being the field operator associated with the spin component $j$. For symmetric systems, we employ the fact that these correlation functions are built up by the same set of orthogonal eigenfunctions [10]. Such eigenfunctions can be interpreted as natural pairing orbits that form the BCS wave functions in inhomogeneous systems. We note that the standard textbook description of BCS wave function refers to infinite homogeneous systems such that each pairing orbit corresponds to the eigenfunctions of opposite momenta [8]. However, the presence of a confining potential in inhomogeneous systems could drastically alter the pairing orbits. Although the time-reversal symmetry helps to fix a certain set of quantum numbers in pairs, the exact form of pairing orbits are difficult to find in general. One possible solution is to treat the pairing orbits as (unknown) variational functions. With the usual BCS ansatz and variation technique, a set of nonlinearly coupled equations of pairing modes have been derived [10, 11]. However, these nonlinear equations have no simple physical interpretations, and numerical solutions

have only been demonstrated in nuclear systems [10, 11]. As the particle numbers in Fermi gases systems are typically much larger than that in nuclei, numerical method based on variational method could become difficult. To our knowledge, natural orbits of Cooper pairs in a trapped Fermi atomic gases have not been fully explored. 
One of the main purposes of our paper is to indicate an efficient way of determining pairing orbits, directly from the two-point correlation functions obtained by BdG mean field equations. To illustrate our method, we will examine the gas in a spherical harmonic trap. For such systems, Bruun and Heiselberg have provided useful insight about the approximate forms of pairing orbits with a different approach [12, 13]. Here, we will present numerically exact examples of pair orbits. With these pairing orbits we can further construct and study the spatial wave function of a Cooper pair. In addition, we will address the quantum entanglement between two constituent atoms in a pair. Recently, one of us have addressed the issue of quantum entanglement of two atoms due to $s$-wave scattering [14]. The study of a Cooper pair would shed some light on the importance of many-body effects.

\section{THEORY}

\section{A. The model and BdG equations}

To begin with, we write down the model Hamiltonian describing an interacting twocomponent Fermi gas in a trapping potential $U_{0}(\mathbf{r})$,

$$
\begin{aligned}
H=\int d^{3} \mathbf{r} & {\left[\psi_{\alpha}^{\dagger}(\mathbf{r}) H_{0} \psi_{\alpha}(\mathbf{r})+\psi_{\beta}^{\dagger}(\mathbf{r}) H_{0} \psi_{\beta}(\mathbf{r})\right] } \\
& +g \int d^{3} \mathbf{r} \psi_{\alpha}^{\dagger}(\mathbf{r}) \psi_{\beta}^{\dagger}(\mathbf{r}) \psi_{\beta}(\mathbf{r}) \psi_{\alpha}(\mathbf{r})
\end{aligned}
$$

where $H_{0}=-\frac{\hbar^{2}}{2 m} \nabla^{2}+U_{0}(\mathbf{r})-\mu$, with $m$ and $\mu$ being the particle mass and chemical potential respectively. The coupling strength $g$ is related to the $s$-wave scattering length $a$ via $g=4 \pi \hbar^{2} a / m$. In this paper we assume that $a$ is negative and the number of atoms in each component is the same.

Under the mean field approximation, the Hamiltonian can be diagonalized through the Bogoliubov transformation: $\psi_{\alpha}(\mathbf{r})=\sum_{\eta} u_{\eta}(\mathbf{r}) \gamma_{\eta \alpha}-v_{\eta}^{*}(\mathbf{r}) \gamma_{\eta \beta}^{\dagger}$, and $\psi_{\beta}(\mathbf{r})=\sum_{\eta} u_{\eta}(\mathbf{r}) \gamma_{\eta \beta}+$ $v_{\eta}^{*}(\mathbf{r}) \gamma_{\eta \alpha}^{\dagger}$, such that the ground state is the vacuum state of the quasi-particle operators $\gamma_{\eta}$ 's, i.e., Bogoliubov vacuum. The quasi-particle wave functions $u_{\eta}(\mathbf{r})$ and $v_{\eta}(\mathbf{r})$ are solved self-consistently by the Bogoliubov-deGennes (BdG) equations [8]:

$$
\begin{aligned}
{\left[H_{0}(\mathbf{r})+W(\mathbf{r})\right] u_{\eta}(\mathbf{r})+\Delta(\mathbf{r}) v_{\eta}(\mathbf{r}) } & =E_{\eta} u_{\eta}(\mathbf{r}) \\
\Delta^{*}(\mathbf{r}) u_{\eta}(\mathbf{r})-\left[H_{0}(\mathbf{r})+W(\mathbf{r})\right] v_{\eta}(\mathbf{r}) & =E_{\eta} v_{\eta}(\mathbf{r})
\end{aligned}
$$


with $W(\mathbf{r})=g \sum_{\eta}\left|v_{\eta}(\mathbf{r})\right|^{2}$, and $\Delta(\mathbf{r})=-g \sum_{\eta} u_{\eta}(\mathbf{r}) v_{\eta}^{*}(\mathbf{r})$. It is important to note that $\Delta(\mathbf{r})$ has a $1 / r$ divergence [7, 15]. Such a divergence can be eliminated by replacing $g$ with a regularized effective coupling constant as described in Ref. [15, 16].

\section{B. Two-point correlation functions}

Given that the ground state of the system is described by the Bogoliubov quasi-particle vacuum, the normal density matrix $\rho\left(\mathbf{r}_{1}, \mathbf{r}_{2}\right)=\left\langle\psi_{\alpha}^{\dagger}\left(\mathbf{r}_{1}\right) \psi_{\alpha}\left(\mathbf{r}_{2}\right)\right\rangle=\left\langle\psi_{\beta}^{\dagger}\left(\mathbf{r}_{1}\right) \psi_{\beta}\left(\mathbf{r}_{2}\right)\right\rangle$ and the anomalous density matrix $\nu\left(\mathbf{r}_{1}, \mathbf{r}_{2}\right)=\left\langle\psi_{\beta}\left(\mathbf{r}_{1}\right) \psi_{\alpha}\left(\mathbf{r}_{2}\right)\right\rangle$ take the form:

$$
\begin{aligned}
& \rho\left(\mathbf{r}_{1}, \mathbf{r}_{2}\right)=\sum_{\eta} v_{\eta}\left(\mathbf{r}_{1}\right) v_{\eta}^{*}\left(\mathbf{r}_{2}\right) \\
& \nu\left(\mathbf{r}_{1}, \mathbf{r}_{2}\right)=-\sum_{\eta} u_{\eta}\left(\mathbf{r}_{1}\right) v_{\eta}^{*}\left(\mathbf{r}_{2}\right) .
\end{aligned}
$$

In this paper we assume that $\rho\left(\mathbf{r}_{1}, \mathbf{r}_{2}\right)$ and $\nu\left(\mathbf{r}_{1}, \mathbf{r}_{2}\right)$ are real symmetric matrices. Such a symmetric property can be shown explicitly in spherically trapped systems that we will discussed later in the paper.

From the properties of quasi-particle wave functions, it can be shown that $\rho$ and $\nu$ commute (see Appendix), i.e.,

$$
\int d^{3} r_{1}\left[\rho\left(\mathbf{r}, \mathbf{r}_{1}\right) \nu\left(\mathbf{r}_{1}, \mathbf{r}_{2}\right)-\nu\left(\mathbf{r}, \mathbf{r}_{1}\right) \rho\left(\mathbf{r}_{1}, \mathbf{r}_{2}\right)\right]=0 .
$$

This implies that the normal density matrix and the anomalous density matrix share a common set of eigenfunctions defined by the integral equations:

$$
\begin{aligned}
& \int d^{3} r_{2} \rho\left(\mathbf{r}_{1}, \mathbf{r}_{2}\right) f_{n}\left(\mathbf{r}_{2}\right)=\lambda_{n} f_{n}\left(\mathbf{r}_{1}\right) \\
& \int d^{3} r_{2} \nu\left(\mathbf{r}_{1}, \mathbf{r}_{2}\right) f_{n}\left(\mathbf{r}_{2}\right)=\chi_{n} f_{n}\left(\mathbf{r}_{1}\right)
\end{aligned}
$$

where $\lambda_{n}$ and $\chi_{n}$ are real eigenvalues, and $\left\{f_{n}\right\}$ is a complete and orthogonal set of eigenfunctions. Furthermore, $\rho\left(\mathbf{r}_{1}, \mathbf{r}_{2}\right)$ and $\nu\left(\mathbf{r}_{1}, \mathbf{r}_{2}\right)$ can be expressed as the following bilinear expansion:

$$
\begin{aligned}
\left\langle\psi_{\alpha}^{\dagger}\left(\mathbf{r}_{1}\right) \psi_{\alpha}\left(\mathbf{r}_{2}\right)\right\rangle & =\sum_{n} \lambda_{n} f_{n}\left(\mathbf{r}_{1}\right) f_{n}^{*}\left(\mathbf{r}_{2}\right) \\
\left\langle\psi_{\beta}\left(\mathbf{r}_{1}\right) \psi_{\alpha}\left(\mathbf{r}_{2}\right)\right\rangle & =\sum_{n} \chi_{n} f_{n}\left(\mathbf{r}_{1}\right) f_{n}^{*}\left(\mathbf{r}_{2}\right)
\end{aligned}
$$

Here the convergence is ensured by the squared integrable property of the kernels, according to the theory of integral equations. 


\section{Natural orbits}

The eigenfunctions $f_{n}$ are called natural orbits. To interpret such orbits, let us write down a general mode expansion of operators $\psi_{\alpha}$ and $\psi_{\beta}$ such that: $\psi_{\alpha}(\mathbf{r})=\sum_{n} \phi_{n}^{(\alpha)}(\mathbf{r}) \alpha_{n}$, $\psi_{\beta}(\mathbf{r})=\sum_{n} \phi_{n}^{(\beta)}(\mathbf{r}) \beta_{n}$, where $\left\{\phi_{n}^{(\alpha)}\right\}$ and $\left\{\phi_{n}^{(\beta)}\right\}$ are two sets of complete orthogonal functions to be determined, and $\alpha_{n}$ and $\beta_{n}$ are the corresponding fermion annihilation operators. Next we construct the BCS ground state in the standard form:

$$
|\Phi\rangle=\prod_{n}\left(\tilde{u}_{n}+\tilde{v}_{n} \alpha_{n}^{\dagger} \beta_{n}^{\dagger}\right)|0\rangle
$$

with the normalized coefficients $\tilde{u}_{n}$ and $\tilde{v}_{n}$ satisfying $\left|\tilde{u}_{n}\right|^{2}+\left|\tilde{v}_{n}\right|^{2}=1$. From the fact that

$$
\begin{gathered}
\left\langle\Phi\left|\psi_{\alpha}^{\dagger}\left(\mathbf{r}_{1}\right) \psi_{\alpha}\left(\mathbf{r}_{2}\right)\right| \Phi\right\rangle=\sum_{n} \phi_{n}^{(\alpha) *}\left(\mathbf{r}_{1}\right) \phi_{n}^{(\alpha)}\left(\mathbf{r}_{2}\right)\left|\tilde{v}_{n}\right|^{2} \\
\left\langle\Phi\left|\psi_{\beta}\left(\mathbf{r}_{1}\right) \psi_{\alpha}\left(\mathbf{r}_{2}\right)\right| \Phi\right\rangle=\sum_{n} \phi_{n}^{(\beta)}\left(\mathbf{r}_{1}\right) \phi_{n}^{(\alpha)}\left(\mathbf{r}_{2}\right) \tilde{u}_{n}^{*} \tilde{v}_{n}
\end{gathered}
$$

we immediately see that the constructed BCS ground state (10) is consistent with the BdG results Eq. (8) and (91) if

$$
\begin{aligned}
& \phi_{n}^{(\alpha)}(\mathbf{r})=f_{n}^{*}(\mathbf{r}) \\
& \phi_{n}^{(\beta)}(\mathbf{r})=f_{n}(\mathbf{r})
\end{aligned}
$$

and $\tilde{v}_{n}=\sqrt{\lambda}_{n}$ and $\tilde{u}_{n}=\chi_{n} / \sqrt{\lambda}_{n}$ (Appendix). In other words, $f_{n}$ and its conjugate are indeed the pairing modes needed for the construction of the BCS ground state. This is shown by matching the correlation functions obtained from BdG mean-field equations. In fact, with the help of Wick's theorem, the choice of mode functions (13) and (14) also match higher order correlation functions. Hence the equivalence between Bogoliubov vacuum and the BCS ground state in a trapped system can be established explicitly.

Historically, the concept of natural orbits were applied to pairing problems of nucleons and one can find some early references in Ref. [10]. Here we employ such an idea to analyze the structure of Cooper pairs in atomic systems. We should emphasize that the key to the existence of $f_{n}$ is the symmetric property of $\rho$, which makes $\rho$ and $\nu$ commute. In the appendix we indicate this point in a simple derivation of Eq. (15). For asymmetric systems, such as the systems with imbalance populations of the two components, $\rho$ and $\nu$ do not commute in general. This would then forbid the ground state to be in the BCS form, although the BdG equations could still be used to describe asymmetric systems. 


\section{Cooper pair wave function and quantum entanglement}

The description of a Cooper pair is inherited from the mean field description, where every pair in the system is assumed to be identical. Let us now construct the wave function of a Cooper pair based on the pairing orbits. This is achieved by noting that the BCS state takes the form:

$$
|\Phi\rangle \propto \exp \left(\sum_{j} \kappa_{j} \alpha_{j}^{\dagger} \beta_{j}^{\dagger}\right)|0\rangle=\sum_{k=0}^{\infty} \frac{A^{\dagger k}}{k !}|0\rangle
$$

where $\kappa_{j}=\tilde{v}_{j} / \tilde{u}_{j}, A^{\dagger}=\sum_{j} \kappa_{j} \alpha_{j}^{\dagger} \beta_{j}^{\dagger}$, and $j$ stands for quantum numbers collectively. The wave function corresponding to the pair creation operator $A^{\dagger}$ is given by

$$
F\left(\mathbf{r}_{1}, \mathbf{r}_{2}\right)=C \sum_{j} \kappa_{j} f_{j}\left(\mathbf{r}_{1}\right) f_{j}^{*}\left(\mathbf{r}_{2}\right)
$$

where $C$ is a normalization constant. Such a function is interpreted as the wave function of a Cooper pair. One reasoning is based on the fact that the BCS state is dominated by particle numbers near the mean value $\bar{N}$ when the particle number is large, i.e., $k \approx \bar{N}$ terms in Eq. (15) contribute most. This allows us to have an approximate picture of $\bar{N}$ pairs described by $A^{\dagger \bar{N}}|0\rangle$. It is worth noting that each term in expansion (16) is weighted by the factor $\kappa_{j}$, which is different from Eq. (8) and (9). Since $\kappa_{j}$ is larger for the more filled orbits, orbits below the Fermi level could have more contributions individually.

Eq. (16) is precisely a form of Schmidt decomposition of a bipartite system [17]. This is observed by the fact that $f_{j}$ are orthogonal basis functions, and $\kappa_{j}$ are corresponding Schmidt eigenvalues. Schmidt decomposition provides a useful way to characterize quantum entanglement between two subsystems in pure states. In particular the degree of quantum entanglement can be learned by the von Neumann entropy according to the distribution of Schmidt eigenvalues. Here we apply this method to address the entanglement between two atoms in a Cooper pair. A transparent and direct measure of entanglement is the 'average' number of Schmidt modes involved. The effective Schmidt number $K$ provides this average 14, 19]:

$$
K=1 / \sum_{j}\left|\kappa_{j}^{\prime}\right|^{4}
$$

where $\kappa_{j}^{\prime}=C \kappa_{j}$. Such a quantity is also the inverse of the purity function. The larger the value of $K$, the higher the entanglement. Note that the entanglement here refers to the spatial degrees of freedom, which should be distinguished from the spin entanglement [18]. 


\section{FERMI GASES IN A SPHERICAL HARMONIC POTENTIAL}

Having described the formalism, we now proceed to study natural orbits of a Fermi gas confined in a spherical harmonic potential: $U_{0}(r)=\frac{1}{2} m \omega^{2} r^{2}$, with $\omega$ being the trapping frequency. First, we determine the quasi-particle mode functions $u_{\eta}$ 's and $v_{\eta}$ 's numerically from the BdG equations. The spherical symmetry of the system gives: $r u_{\eta l m}(\mathbf{r})=u_{\eta l}(r) Y_{l m}(\theta, \phi)$, $r v_{\eta l m}(\mathbf{r})=v_{\eta l}(r) Y_{l m}(\theta, \phi)$, with $Y_{l m}$ being the spherical harmonic functions. It should be noted that in order to remove the ultraviolet divergence, we employ an effective coupling constant $g_{\text {eff }}(\mathbf{r})$, such that the whole set of equations is independent on cutoff. The formulation of renormalization scheme has been discussed extensively in [15, 16], and we will skip the details in this paper.

Once numerical solutions of $u_{\eta}$ 's and $v_{\eta}$ 's are found, the correlation functions $\rho\left(\mathbf{r}_{1}, \mathbf{r}_{2}\right)$ and $\nu\left(\mathbf{r}_{1}, \mathbf{r}_{2}\right)$ are obtained from Eqs. (3) and (4). A significant simplification can be made by using the addition formula: $(2 l+1) P_{l}(\cos \gamma)=4 \pi \sum_{m=-l}^{l} Y_{l m}^{*}\left(\theta_{1}, \phi_{1}\right) Y_{l m}\left(\theta_{2}, \phi_{2}\right)$, where $P_{l}(\cos \gamma)$ is the Legendre polynomial, and $\gamma$ is the angle between between $\mathbf{r}_{1}$ and $\mathbf{r}_{2}$. This gives

$$
\begin{aligned}
& \left\langle\psi_{\alpha}^{\dagger}\left(\mathbf{r}_{1}\right) \psi_{\alpha}\left(\mathbf{r}_{2}\right)\right\rangle=\sum_{l} P_{l}(\cos \gamma) \alpha_{l}\left(r_{1}, r_{2}\right) \\
& \left\langle\psi_{\beta}\left(\mathbf{r}_{1}\right) \psi_{\alpha}\left(\mathbf{r}_{2}\right)\right\rangle=-\sum_{l} P_{l}(\cos \gamma) \beta_{l}\left(r_{1}, r_{2}\right)
\end{aligned}
$$

where

$$
\begin{aligned}
& \alpha_{l}\left(r_{1}, r_{2}\right)=\frac{2 l+1}{4 \pi} \sum_{\eta} \frac{v_{\eta l}\left(r_{1}\right) v_{\eta l}^{*}\left(r_{2}\right)}{r_{1} r_{2}} \\
& \beta_{l}\left(r_{1}, r_{2}\right)=\frac{2 l+1}{4 \pi} \sum_{\eta} \frac{u_{\eta l}\left(r_{1}\right) v_{\eta l}^{*}\left(r_{2}\right)}{r_{1} r_{2}} .
\end{aligned}
$$

Therefore the correlation functions (3) and (41) depends only on three variables: $r_{1}, r_{2}$ and $\gamma$. The integral eigenvalue equations (6) and (7), can be solved conveniently by expressing the eigenfunctions as $f_{n l m}(\mathbf{r})=\frac{1}{r} h_{n l}(r) Y_{l m}(\theta, \phi)$, with $h_{n l}(r)$ being the radial function associated with the radial quantum number $n$ and orbital angular momentum $l$. Equations (6) and (7) then become,

$$
\begin{aligned}
& \int r_{1} r_{2} \alpha_{l}\left(r_{1}, r_{2}\right) h_{n l}\left(r_{2}\right) d r_{2}=\left|\tilde{v}_{n l}\right|^{2} h_{n l}\left(r_{1}\right) \\
& \int r_{1} r_{2} \beta_{l}\left(r_{1}, r_{2}\right) h_{n l}\left(r_{2}\right) d r_{2}=\tilde{u}_{n l}^{*} \tilde{v}_{n} h_{n l}\left(r_{1}\right)
\end{aligned}
$$




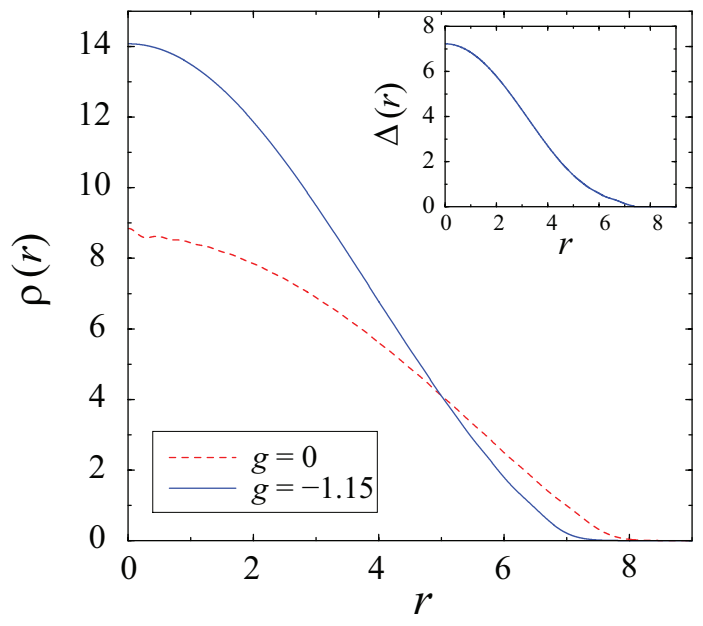

FIG. 1: (Color online) The $g=-1.15$ (solid line) and the non-interacting (dashed line) radial density distribution function, normalized to $N=\int 4 \pi r^{2} \rho(r) d r$. Inset shows the pairing field $\Delta(r)$ with $g=-1.15$.

which are one-dimensional integral equations, as only the radial coordinates are involved.

To provide a concrete example, we consider a system of $N=N_{\alpha}+N_{\beta}=2 N_{\alpha}=10912$ particles with the interaction strength $g=-1.15$ in trap units, (i.e., energy in $\hbar \omega$, length in $\sqrt{\hbar / m \omega})$. The choice of such a particle number corresponds to the Fermi energy $\epsilon_{F}=31.5 \hbar \omega$ in the non-interacting limit. Eq.(2) was solved in a truncated harmonic oscillator states basis, with a cutoff energy $\sim 180 \hbar \omega \gg \mu$. To achieve convergent results, we employed the regularization scheme according to Ref. [16]. The particle density $\rho(r)$ and pairing potential $\Delta(r)$ are consistently solved and shown in Fig 1. In this example, $\Delta(0)=7.2 \hbar \omega$ represents a modest strong coupling. Comparing with the non-interacting system (dashed line), we see that the particles are significantly dragged towards the center of the trap due to the attractive interaction.

After solving the integral eigenvalue equations for $h_{n l}$, we obtain the distributions of eigenvalues $\left|\tilde{v}_{n l}\right|^{2}$ and $\tilde{u}_{n l}^{*} \tilde{v}_{n l}$ for various angular momentum quantum number $l$. We note that in the case of non-interacting systems at zero temperature, $\left|\tilde{v}_{n l}^{2}\right|$ is a step function, i.e., $\left|\tilde{v}_{n l}\right|^{2}=\Theta\left(\mu-\frac{3}{2}-l-2 n\right)$. This corresponds to the fact that non-interacting atoms fill up all the trap energy states up to the Fermi level. For the interacting system considered here, the sharp edge of the step function is smeared out as shown in Fig. 2a. Such a smearing effect is similar to what appears in uniform BCS systems [8]. However, the difference here 

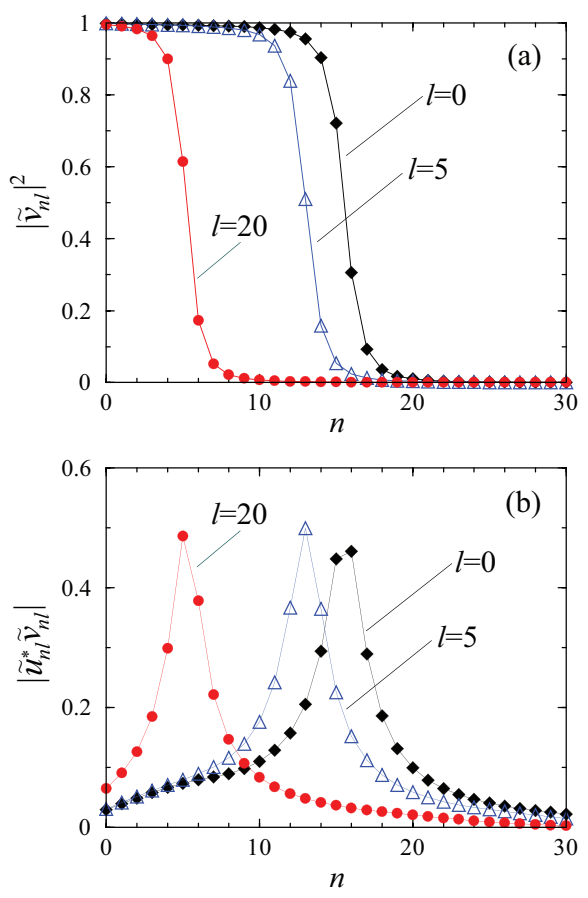

FIG. 2: (Color online) Occupation number $\left|\tilde{v}_{k l}\right|^{2}$ (upper panel) and the pairing amplitude $\tilde{u}_{n l} \tilde{v}_{n l}$ (lower panel) with $l=0$ (diamond), 5 (triangle), 20 (circle). Same parameters as in Fig. 1.

is that $f_{n l}$ are now the pairing basis instead of plane waves.

In Fig. 2b, we show the quantity $\tilde{u}_{n l}^{*} \tilde{v}_{n l}$, which measures the coherence between occupied and un-occupied states. We see that $\tilde{u}_{n l}^{*} \tilde{v}_{n l}$ reaches a peak at a certain values of radial quantum number $n$. These $n$ 's correspond to orbits $f_{n l}$ that have average energies close to the chemical potential, and hence for higher $l$, the peak appears at lower $n$. The orbits near the peak contribute most significantly to the gap function. The shapes of some of these orbits are plotted in Fig. 3] where the radial part $\left|h_{n l}(r)\right|^{2}$ at various quantum numbers $l$ is shown. Comparing with bare eigenfunctions of the trap (dashed line) with the same quantum numbers $n$ and $l$, we observe similar oscillatory patterns but the envelopes are more concentrated towards the trap center. This indicates that the trap's eigenfunctions do not provide a good approximation to the actual pairing orbits, at least for the moderate strong coupling considered here. However, for a much weaker coupling $\Delta(0) \ll \hbar \omega$ (not shown), we do find a good agreement between pairing orbits and the bare trap's eigenfunctions near the Fermi surface, which is expected according to the argument in Ref. 12].

In Fig. 4. we illustrate the shape of the Cooper pair wave function by plotting the quantity $P=\left|r_{1} r_{2} F\left(r_{1}, r_{2}, \gamma\right)\right|^{2}$ at various angular separation $\gamma$. Note that $P$ is proportional 

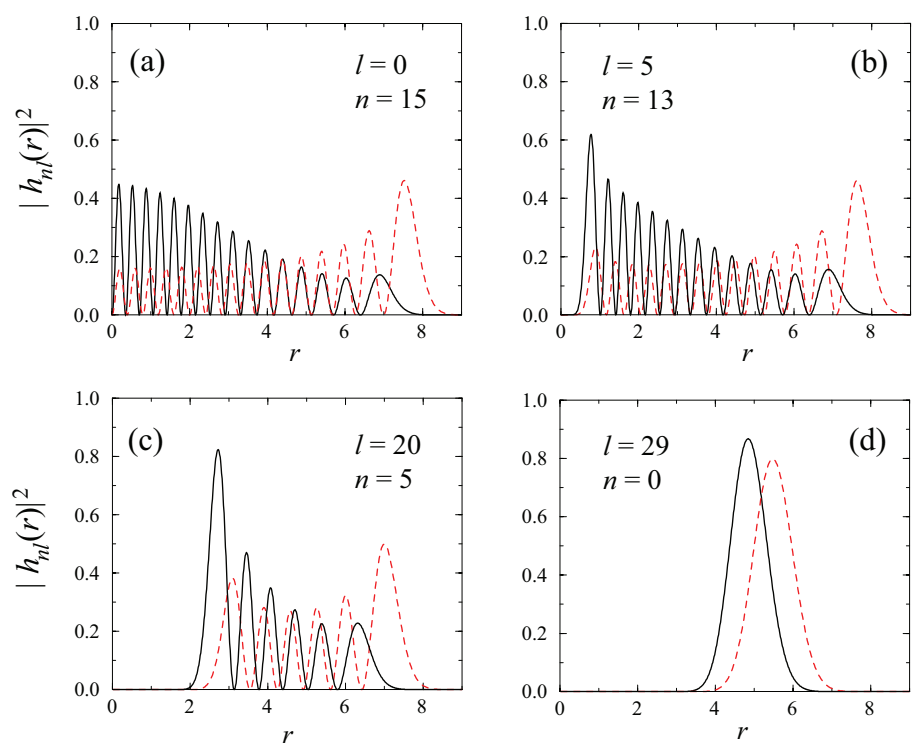

FIG. 3: (Color online) Some radial mode functions $\left|h_{n l}(r)\right|^{2}$ of natural orbits, normalized to $\int\left|h_{n l}(r)\right|^{2} d r=1$, with $g=-1.15$ (solid line). These functions correspond to those pairing orbits with a peak value of $\tilde{u}_{n l} \tilde{v}_{n l} \sim 0.5$ at a given $l$. The dashed lines correspond to the bare harmonic trap radial wave functions having the respective quantum numbers.

to probability density of simultaneously finding the two particles at $\mathbf{r}_{1}$ and $\mathbf{r}_{2}$, and the weighting factor $r_{1} r_{2}$ is included for spherical systems. In both Fig. 4a and Fig. 4b, we see some interesting fringes patterns, but the main feature is that the function $P$ appears to be localized near the diagonal $r_{1}=r_{2}$, indicating that both atoms are likely to be found in the same radial distance from the trap center. In addition, by comparing Fig. 4a and 4b, $P$ drops significantly when angular separation $\gamma$ increases. This suggests that $F\left(r_{1}, r_{2}, \gamma\right)$ mainly concentrates at $\mathbf{r}_{1}=\mathbf{r}_{2}$, not just the same radial distance. For the example shown in Fig. 4a, the width of $P$ near the peak is about 1.2 which is comparable to the bare trap ground state.

The effective Schmidt number $K$ defined in Eq. (17) is found to be $K \approx 1749$, which is roughly the same order of particle number in the trap of this example. It is useful to compare this number with the value of $K$ of a two-atom system with the same scattering length. According to the calculation in Ref. [14], a two-atom system in the ground state has a very weak entanglement if the scattering length is small compared with the trap length unit. For the parameters used in Fig. 4, the scattering length is $a=-0.09$ which gives $K \approx 1$ in the two-atom system. Therefore a Cooper pair, which is due to many-body effects, 
(a) $\gamma=0$
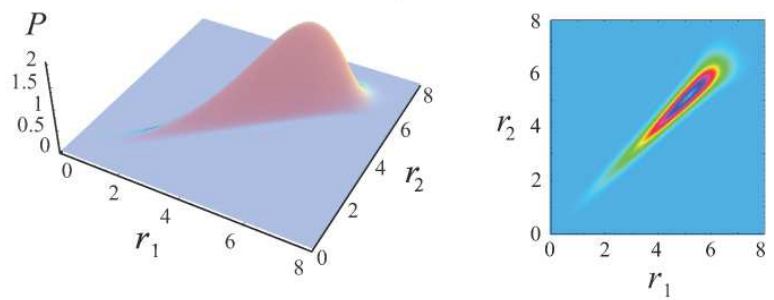

(b) $\gamma=\pi / 20$

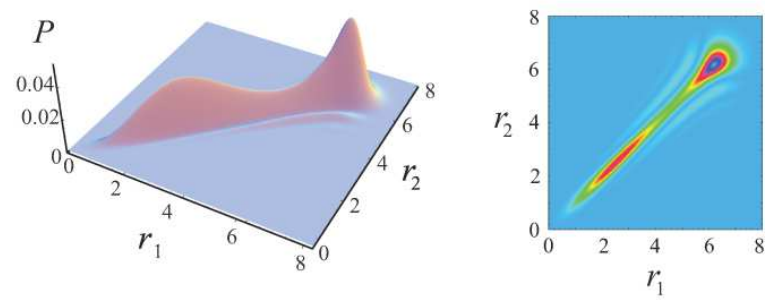

FIG. 4: (Color online) An illustration of the spatial profile of a Cooper pair, where $P=\left|r_{1} r_{2} F\left(r_{1}, r_{2}, \gamma\right)\right|^{2}$, with (a) $\gamma=0$ and (b) $\gamma=\pi / 20$. Same parameters as in Fig. 1.

processes a much stronger entanglement.

As a final remark, we note in Ref. [20] that the ratio $K / N_{j}$ (where $N_{\alpha}=N_{\beta}$ is particle number of a component) is suggested to be an indicator of determining whether a composite two-particle system behave as a boson or not. Specifically, $K / N \gg 1$ is the regime where composite particles can be described by creation and annihilation operators obeying bosonic commutation relations. Here in our example, we have $K / N \approx 1749 / 5456$ which is still smaller than one. Therefore the entanglement is not strong enough to hold the Cooper pair together as a boson.

\section{CONCLUSIONS}

To summarize, eigen-mode expansion of correlation functions provides a powerful tool to reveal the underlying coherent structures. Such a strategy is also known useful in various areas of physics, such as nuclear physics and optics [21]. In this paper, we have examined the eigenfunctions of two-point correlation functions (3) and (4) that can be obtained from the solutions of BdG equations. These eigenfunctions correspond to natural pairing orbits in the BCS state (10), and they serve as Schmidt basis vectors for the construction of Cooper pair wave functions. Hence, one can further analyze the quantum entanglement associated 
with the spatial degree of freedom. We have demonstrated the method in a spherically trapped system, in which the natural orbits are calculated explicitly. In particular, our numerical results indicate features that reflect the strong quantum entanglement between two constituent atoms in a pair. It is useful to point out that the method could also be extended to BEC-BCS crossover problems, where BdG equations are modified by the molecular field and a hybrid form of BCS-molecule state may be constructed. The structure of natural orbits in this regime is a very interesting topic for future investigations.

\section{Acknowledgement}

This work is supported in part by the Research Grants Council of the Hong Kong Special Administrative Region, China (Project No. 401305 and 400504).

\section{APPENDIX}

The vanishing commutator given in Eq. (5) can be derived under a rather general consideration (see for example, in Ref. [10]). Here we re-derive the result in a more transparent way. We start with the orthogonality and completeness relations of the quasi-particle wave functions [8]

$$
\begin{array}{r}
\int d^{3} r\left[u_{m}^{*}(\mathbf{r}) u_{n}(\mathbf{r})+v_{m}^{*}(\mathbf{r}) v_{n}(\mathbf{r})\right]=\delta_{m n} \\
\sum_{\eta} v_{\eta}\left(\mathbf{r}_{1}\right) v_{\eta}^{*}\left(\mathbf{r}_{2}\right)+u_{\eta}^{*}\left(\mathbf{r}_{1}\right) u_{\eta}\left(\mathbf{r}_{2}\right)=\delta^{3}\left(\mathbf{r}_{1}-\mathbf{r}_{2}\right)
\end{array}
$$

Together with Eqs. (3) and (41), we can show

$$
\begin{aligned}
& \int d^{3} r_{1} \rho\left(\mathbf{r}_{1}, \mathbf{r}\right) \nu\left(\mathbf{r}_{1}, \mathbf{r}_{2}\right) \\
= & \nu\left(\mathbf{r}, \mathbf{r}_{2}\right)+\sum_{m n} \int d^{3} r_{1} u_{m}^{*}\left(\mathbf{r}_{1}\right) u_{m}(\mathbf{r}) u_{n}\left(\mathbf{r}_{1}\right) v_{n}^{*}\left(\mathbf{r}_{2}\right) \\
= & \nu\left(\mathbf{r}, \mathbf{r}_{2}\right)+\sum_{m n} u_{m}(\mathbf{r}) v_{n}^{*}\left(\mathbf{r}_{2}\right)\left(\delta_{m n}-\int d^{3} r_{1} v_{m}^{*}\left(\mathbf{r}_{1}\right) v_{n}\left(\mathbf{r}_{1}\right)\right) \\
= & -\int d^{3} r_{1} \sum_{m n} u_{m}(\mathbf{r}) v_{m}^{*}\left(\mathbf{r}_{1}\right) v_{n}^{*}\left(\mathbf{r}_{2}\right) v_{n}\left(\mathbf{r}_{1}\right) \\
= & \int d^{3} r_{1} \nu\left(\mathbf{r}, \mathbf{r}_{1}\right) \rho\left(\mathbf{r}_{1}, \mathbf{r}_{2}\right) .
\end{aligned}
$$


Now we see that Eq. (5) holds if the symmetric condition: $\rho\left(\mathbf{r}_{1}, \mathbf{r}_{2}\right)=\rho\left(\mathbf{r}_{2}, \mathbf{r}_{1}\right)$ is satisfied. With Eqs. (24) and (25) and the symmetric $\rho\left(\mathbf{r}_{1}, \mathbf{r}_{2}\right)$, we also have the relation:

$$
\int \rho\left(\mathbf{r}, \mathbf{r}_{1}\right) \rho\left(\mathbf{r}_{1}, \mathbf{r}_{2}\right)+\nu^{*}\left(\mathbf{r}, \mathbf{r}_{1}\right) \nu\left(\mathbf{r}_{1}, \mathbf{r}_{2}\right) d^{3} r_{1}=\rho\left(\mathbf{r}, \mathbf{r}_{2}\right)
$$

Projecting both sides of Eq. (27) onto $f_{n}\left(\mathbf{r}_{2}\right)$, we obtain a relation of the eigenvalues,

$$
\lambda_{n}+\chi_{n}^{2} / \lambda_{n}=1
$$

which is consistent with the normalization condition $\left|\tilde{u}_{n}\right|^{2}+\left|\tilde{v}_{n}\right|^{2}=1$ of BCS wave function.

[1] H. T. C. Stoof et al., Phys. Rev. Lett. 76, 10 (1996).

[2] B. DeMarco and D. S. Jin, Science 285, 1703 (1999); A. C. Truscott et al., Science 291, 2570 (2001); Z. Hadzibabic, Phys. Rev. Lett. 88, 160401 (2002).

[3] C. Chin et al., Science 305, 1128 (2004).

[4] M. W. Zwierlein et al., Phys. Rev. Lett. 92, 120403 (2004); M. W. Zwierlein et al., Nature 435, 1047 (2005).

[5] S. Gupta et al., Science 300, 1723 (2004).

[6] M. Greiner, C. A. Regal, J. T. Stewart, and D. S. Jin, Phys. Rev. Lett. 94, 110401 (2005).

[7] G. M. Bruun et al., Eur. Phys. J. D 9, 433 (1999).

[8] P. G. de Gennes, Superconductivity of Metals and Alloys (W.A. Benjamin INC., 1966); J. B. Ketterson and S. N. Song, Superconductivity (New York, Cambridge University Press, 1999).

[9] S. Folling et al., Nature (London) 434, 481 (2005); L.-M. Duan, Phys. Rev. Lett. 96, 103201 (2006).

[10] J. Dobaczewski, W. Nazarewicz, T. R. Werner, J. F. Berger, C. R. Chinn, and J. Decharg, Phys. Rev. C 53, 2809 (1996) and references therein.

[11] P.-G. Reinhard, M. Bender, K. Rutz, J. A. Maruhn, Z. Phys. A 358, 277 (1997).

[12] G. M. Bruun and H. Heiselberg, Phys. Rev. A 65, 053407 (2002).

[13] H. Heiselberg and B. Mottelson Phys. Rev. Lett. 88, 190401 (2002); H. Heiselberg, Phys. Rev. A 68, 053616 (2003).

[14] J. Wang, C. K. Law, M. C. Chu, Phys. Rev. A 72, 022346 (2005).

[15] A. Bulgac and Y. Yu, Phys. Rev. Lett. 88, 042504 (2002). 
[16] M. Grasso and M. Urban, Phys. Rev. A 68, 033610 (2003).

[17] For a review of Schmidt decomposition, see A. Ekert and P. L. Knight, Am. J. Phys. 63, 415 (1995) and references therein.

[18] C. Dunning et al., Phys. Rev. Lett. 94, 227002 (2005).

[19] R. Grobe, K. Rzążewski and J. H. Eberly, J. Phys. B 27, L503 (1994).

[20] C. K. Law, Phys. Rev. A 71, 034306 (2005).

[21] L. Mandel and E. Wolf, Optical coherence and quantum optics, (Cambridge, 1995). 